\title{
Quantitative Comparison of Cone Beam Computed Tomography and Microradiography in the Evaluation of Bone Density after Maxillary Sinus Augmentation: A Preliminary Study
}

\author{
Carlo M. Soardi, MD, DDS;* Davide Zaffe, MBiolSc; ${ }^{\dagger}$ Alessandro Motroni, MEng; ${ }^{\ddagger}$ \\ Hom-Lay Wang, DDS, MSD, $\mathrm{PhD}^{\S}$
}

\begin{abstract}
Purpose: Cone beam computed tomography (CBCT) and microradiographic analyses were comparatively performed in maxillary sinus augmentation to preliminarily verify the diagnostic potential of CBCT on the evaluation of bone regeneration.

Materials and Methods: A two-stage protocol was conducted in 19 consenting patients, all having the crestal bone $\leq 2 \mathrm{~mm}$, in private dental office. Mineralized human bone allograft particles were used to augment sinus using lateral window approach. A succession of CBCT scans of the maxilla was taken before surgery, after sinus augmentation, and immediately after implant insertion. Using virtual probes, CBCT data were processed by medical imaging software and expressed as gray level (GL). A bone core biopsy was taken at implant placement, 6 months after surgery. Microradiography of transverse sections, taken 6,8 , and $10 \mathrm{~mm}$ from the crestal surface, of methacrylate-embedded biopsies was performed to analyze and to evaluate the mineralized material amount (MM\%).
\end{abstract}

Results: A total of 21 sinus augmentations were performed. CBCT (mean GL: 646-693) data were not statistically different when comparing 6-, 8-, and 10-mm sites to after grafting/implant-insertion values. Furthermore, microradiographic (mean MM\%: 45.3-48.3) data were not statistically different comparing 6-, 8-, and 10-mm sites, due to variation of values among patients. A GL and MM\% parallelism was identified considering each patient, instead. A significant correlation $(p<.001)$ between GL and MM\% was found after both Wilcoxon test for paired data and simple linear regression analysis.

Conclusions: The preliminary result clearly demonstrated the predictability of the CBCT analysis. Due to the limited sample and great variations of the MM\% recorded in patients, further clinical and morphometric studies are needed to fulfill diagnostic expectations.

KEY WORDS: bone allograft, cone beam CT, histological analysis, microradiography, sinus augmentation

\footnotetext{
${ }^{\star}$ Private practice, Brescia, BS, Italy; ${ }^{\dagger}$ Department of Biomedical, Metabolic and Neural Sciences, University of Modena and Reggio Emilia, Modena, Italy; ${ }^{\ddagger}$ AMIRG (Applied Medical Imaging Research Group), Milan, Italy; ${ }^{\varsigma}$ Department of Periodontics and Oral Medicine, School of Dentistry, University of Michigan, Ann Arbor, MI, USA
}

Reprint requests: Professor Davide Zaffe, Dipartimento di Scienze Biomediche, Metaboliche e Neuroscienze, Università di Modena e Reggio Emilia, Via del pozzo 71, Policlinico, 41124 Modena, MO, Italy; e-mail: davide.zaffe@unimore.it

(C) 2012 Wiley Periodicals, Inc.

DOI 10.1111/cid.12016

\section{INTRODUCTION}

The axial computer-assisted tomography (computed tomography $[\mathrm{CT}]$ ) allowed a perspective always missing from conventional radiography, i.e., the body sectioning in the third dimension. Besides this important feature, CT can provide quantitative data about the bone mineral density, and this information was promptly correlated with those of other imaging techniques ${ }^{1}$ or bone status. ${ }^{2,3}$ CT scans have been correlated with histomorphometry to discover the CT predictive value on bone density, volume, structures and remodeling, ${ }^{4,5}$ or implant stability. ${ }^{6}$ The introduction of the cone beam 
$\mathrm{CT}$ (CBCT) reduced the scanning time, lowering the irradiation dose and cost, allowing the use of CBCT in dental office as standard instrument. In dentistry, CBCT improved diagnosis and provided additional information for treatment consultation. ${ }^{7}$ Additionally, CBCT could be predictive, like conventional CT, as gray density values for the subjective bone quality classification. ${ }^{8}$

Histomorphometry can be performed on two different histological media: thin, usually 5 to $7 \mu \mathrm{m}$, and thick, about $100 \mu \mathrm{m}$, sections. The first can be performed from paraffin or poly(methyl methacrylate) (PMMA)embedded specimen, but both suffer from artifacts due to sectioning. ${ }^{9}$ Furthermore, stained thick sections might show some trouble as only the surface is stained. Microradiography, also called contact radiography, of thick sections, an analytical way very much liked to CT, might obviate to these problems as it allows the reliable and reproducible investigation of the trabecular architecture of bone on histological media without spatial deformations. ${ }^{9}$ The absence of spatial deformation of microradiographs allowed the quantitative evaluation of the bone mineralization degree ${ }^{10}$ or comparison with CT in reconstruction of critical size bone defects. ${ }^{11}$

On the contrary, the microradiographic analysis was never used to perform comparative studies, aiming the evaluation of CT potentialities. In particular, almost all comparative studies performed the analysis of the grouped CT scan with all collected histomorphometry, ${ }^{4,5}$ but no study was performed comparing CT scan and section microradiography of the same site in the same patient.

The purpose of this work was the correlative analysis of CBCT densitometric data with microradiograph morphometry to verify the diagnostic potential of CBCT on the evaluation of mineral material content after maxillary sinus augmentation.

\section{MATERIALS AND METHODS}

\section{Surgery}

The patients of this study were partially (at least molars and premolars missing) or totally edentulous and needing of either unilateral or bilateral maxillary sinus augmentation. The patients, examined and treated in private dental office, were selected from a pool of subjects, which, using panoramic radiographs, require maxillary sinus augmentation for the placement of delayed posterior implants, and enrolled consecutively.
Additional inclusion criteria were less than $2 \mathrm{~mm}$ of crestal bone height of the sinus floor, as measured on the serial section of the CBCT, good general health, not a smoker, absence of diseases that affect bone metabolism (hyperparathyroidism, osteomalacia, osteoporosis, etc.) or wound healing, ${ }^{12}$ absence of specific disease or problems within the maxillary sinus, and no bisphosphonates or regular medication consumption for more than 3 months. All patients signed their informed consent in which all procedures of the study were detailed, according to the Helsinki protocols. ${ }^{13}$

Surgical sites were infiltrated by local anesthetic (Articain hydrochloride - Ultracain, Sanofi-Aventis Deutschland $\mathrm{GmbH}$, Frankfurt, Germany). A fullthickness flap was reflected to expose the lateral wall of the sinus. A bony window osteotomy ${ }^{14}$ was performed using a piezosurgical device (Mectron S.p.A., Carasco, GE, Italy). The bony window was lifted, without removal, at first wall movement, then the Schneiderian membrane was gently lifted using the piezosurgical device and subsequently a broad curette.

A mixture of cortical and cancellous (80/20) mineralized human bone allograft (MHBA) (Puros, Zimmer Dental Inc., Carlsbad, CA, USA), each consisting of a 50:50 mixture of 0.25 - to $1.0-$ and $1.0-$ to $2.0-\mathrm{mm}$ particles, about $3.0 \pm 0.5 \mathrm{~mL},{ }^{15,16}$ moistened by saline solution, was used as grafting material. ${ }^{17}$ The graft was gently packed on each sinus floor, beneath the membrane. Before soft tissue closure, an absorbable collagen membrane (BioMend ${ }^{\circledR}$, Zimmer Dental Inc.) was placed over the window and the vestibular flap repositioned using surgical Gore-Tex 5/0 (W. L. Gore \& Associates, Flagstaff, AZ, USA). Patients were then treated with amoxicillin (Ratiopharm GmbH, Ulm, Germany) $1 \mathrm{~g}$ twice a day for 6 to 7 days and Synflex forte $550 \mathrm{mg}$ (Recordati SpA, Milano, Italy) as analgesic, if needed, after surgery. Patients were directed to use a chlorhexidine mouthwash $(0.12 \%)$, twice a day, and not to brush the surgical sites for 2 weeks. Sutures were removed 12 to 14 days after the surgery. Monthly, follow-up was scheduled to check for wound dehiscence up to implant insertion.

A bone core specimen was collected before each implant insertion $(3.7$ or $4.7 \mathrm{~mm}$, external diameter, Tapered Screw Vent, Zimmer Dental Inc.), using a trephine assuring an unbroken core biopsy longer than $11 \mathrm{~mm}$ (modified trepan mill, Stoma ${ }^{\circledR} \mathrm{GmbH}$, Emmingen-Liptingen, Germany) under a saline jet at $600 \mathrm{rpm}$. The bone core specimens were collected with 
the assistance of surgical guides that was based upon the individual prosthetic requirements, 6 months after sinus augmentation procedures. As the final position of the drill can greatly vary, ${ }^{18}$ the drilling was performed up to $14 \mathrm{~mm}$ to allow proper length to harvest core specimens. Implant sites were accurately planned to place exactly implants in purely augmented skeletal segments, including possible residual crest. The retrieved bone core samples were immediately washed in sterile saline, gently wiped, labeled on the crestal (periosteal) surface by China (black) ink-pad blotting, then fixed for the microradiographic study.

\section{$\mathrm{CBCT}$}

Apart from the preoperative panoramic radiographs, a succession of CBCT scans (ProMax 3D Max CBVT, Planmeca OY, Helsinki, Finland) of the maxilla was taken for each patient. CBCT was scheduled before surgery, after sinus augmentation, and immediately after implant insertion. The DICOM data were processed with a medical imaging software (3Diagnosys ${ }^{\circledR}$ 3.03DIEMME, Cantù CO, Italy), which gives the possibility to use a virtual probe to extract the bone density values in the desired regions and export them in Excel tables for statistical analysis. The virtual probes had a diameter of 6 or $7 \mathrm{~mm}$ ( $2 \mathrm{~mm}$ wider than planned implants) and were set in regions of the sinus augmentation or implant insertion. Each probe extracts the voxel gray level (GL) data surrounding the region of interest and along the whole direction of the implant; in this way, it is possible to analyze the image GLs correspondent to the selected tissue densities and make a comparison between the different samples. ${ }^{19}$

Bone density values around implants were collected excluding the area affected by artifacts such as photon beam hardening. The data extracted were expressed in GL, as the CBCT system is not calibrated in Hounsfield units (HUs) like a Medical Quantitative CT. This calibration factor is not a limit as all the data have been acquired with the same machine and a comparative analysis has been employed between the different acquisitions. The mean \pm standard deviation of GL was calculated in CBCT sections at 6,8 , and $10 \mathrm{~mm}$ from the crestal surface.

\section{Microradiography}

The cylindrical bone samples were fixed in $4 \%$ paraformaldehyde (all reagents came from Fluka,
Sigma-Aldrich Schweiz, Buchs SG, Switzerland) in $0.1 \mathrm{M}$ phosphate buffer, $\mathrm{pH} 7.2$, for 4 hours at room temperature. Specimens were dehydrated through ethanol series at $4^{\circ} \mathrm{C}$, then embedded in PMMA using a water bath at $4^{\circ} \mathrm{C}$ as described elsewhere. ${ }^{20}$

Using a diamond saw microtome (SP1600, Leica Microsystem, Nussloch, Germany), each PMMAembedded bone sample was transversely sectioned to obtain thick sections $(200 \mu \mathrm{m})$ of the cylindrical sample at 5.9, 7.9, and $9.9 \mathrm{~mm}$ from the crestal (periosteal) surface. The section was reduced to $100 \mu \mathrm{m}$ by grinding, perfectly polished with emery paper and alumina, then $\mathrm{x}$-ray microradiographed (3K5, Italstructures, Riva del Garda TN, Italy) at $15 \mathrm{kV}$ and $10 \mathrm{~mA}$ on high-resolution film (SO 343, Eastman Kodak Co, Rochester, NY, USA).

The microradiographs were analyzed and photographed using a microscope (Axiophot, Carl Zeiss AG, Oberkochen, Germany) under ordinary light. The amount of mineralized material (MM\%), (bone volume $[\mathrm{BV}]+$ graft volume $[\mathrm{GV}]) /$ tissue volume $[\mathrm{TV}]$, was evaluated on the microradiographs using a suitable program for image analyzer and software (AnalySIS ${ }^{\circledR}$, Soft Imaging System GmbH, Münster, Germany).

\section{Statistical Analysis}

Comparisons were performed by means of the nonparametric Kruskal-Wallis test, Wilcoxon test for paired data, and the linear regression analysis. ${ }^{21}$ The null hypothesis $\mathrm{H}_{0}$ was rejected for a critical significance level of $p<.05$.

\section{RESULTS}

\section{Surgery}

Nineteen selected patients, 8 males and 11 females, aged between 36 and 75, mean $=56.8$ years, underwent unilateral $(n=17)$ or bilateral $(n=2)$ maxillary sinus augmentation with a total of 21 treated sinuses. Primary wound closure was obtained in all surgeries and no complaint or adverse effects were observed during the follow-up.

A total of 21 core biopsies (Figure 1) and correspondingly a total of 21 implants were placed in those sites according to individual implant-prosthetictreatment plan, which were obtained from the 19 patients.

\section{CBCT}

$\mathrm{CBCT}$ analyses were regularly performed in each patient before surgery, immediately after sinus grafting, and at 

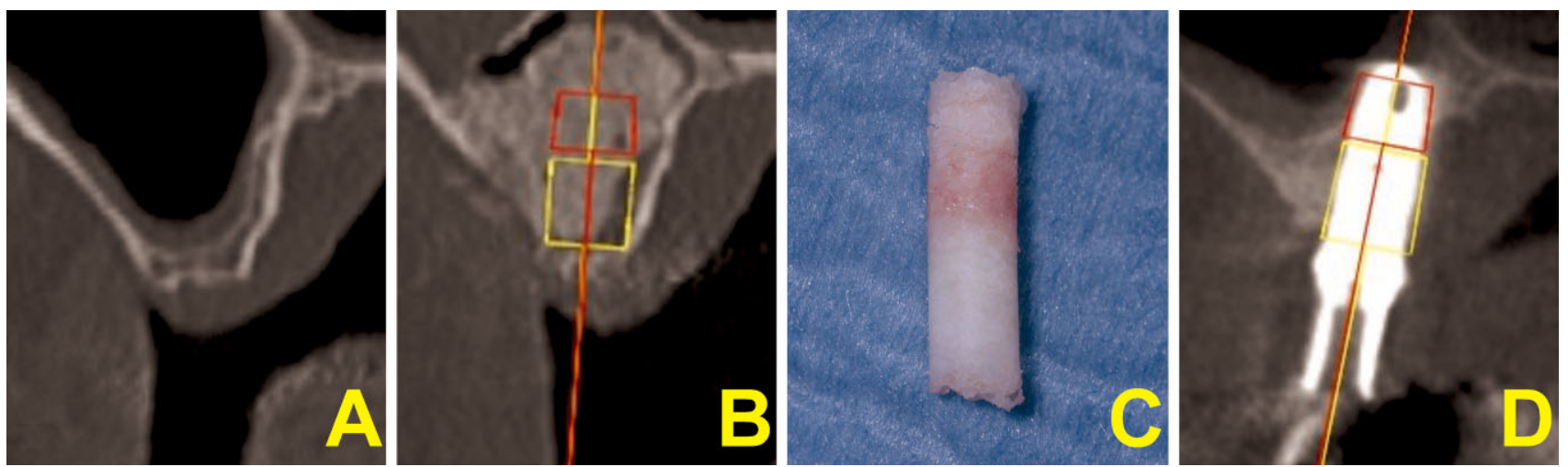

Figure 1 Representative image of G.F. (66-year-old) right-maxillary sinus: anteroposterior CT before (A), immediately after augmentation (B), 12-mm core biopsy after trephining before ink labeling (C), and anteroposterior CT at implant insertion (D). Note the starting minimal amount of crestal bone $(\leq 2 \mathrm{~mm})$. Density evaluations were performed inside the red virtual probe. $\mathrm{CT}=$ computed tomography; G.F. = initials of the patient.

implant insertion (see Figure 1). No remarkable differences in the hard tissue density of each sinus lift were observed at the CBCT visual analysis.

The postgrafting and implant-insertion GL, recorded at 6,8 , and $10 \mathrm{~mm}$ in the 21 sinuses, is summarized in Table 1. The mean GL values ranged between six hundred forty-six and six hundred ninety-three, with maximum values at 8 -mm sites, both after grafting and at implant-insertion times. No great differences existed between mean GL values, both comparing "after grafting" and "at implant-insertion" sites or "after grafting" and "at implant-insertion" corresponding sites (see Table 1).

\section{Microradiography}

Six months after the surgery, the biopsies showed a composite formed by MHBA particles and newly formed bone trabeculae (Figure 2). The majority of MHBA particles were directly connected with the newly formed bone, and very few particles were totally surrounded by the fibrous tissue.

\begin{tabular}{lccc} 
TABLE 1 GLs of the 21 Patient Sites \\
$\begin{array}{l}\text { After Grafting } \\
\text { GL CBCT }\end{array}$ & $p(\mathrm{~W})$ & $\begin{array}{c}\text { At Implant Insertion } \\
\text { GL CBCT }\end{array}$ \\
\hline $6 \mathrm{~mm}$ & $667.0 \pm 272.1$ & .91 & $687.2 \pm 264.0$ \\
$8 \mathrm{~mm}$ & $682.2 \pm 311.3$ & .50 & $693.0 \pm 252.2$ \\
$10 \mathrm{~mm}$ & $663.1 \pm 301.4$ & .50 & $646.7 \pm 282.1$ \\
$p(\mathrm{~K}-\mathrm{W})$ & .87 & & .88
\end{tabular}

Data are expressed as mean \pm standard deviation.

$\mathrm{CBCT}=$ cone beam computed tomography; $\mathrm{GL}=$ gray level; $p(\mathrm{~K}-\mathrm{W})=$ probability after Kruskal-Wallis test; $p(\mathrm{~W})=$ probability after Wilcoxon test for paired data.
The mean MM\% (bone + MBHA), recorded in the section microradiographies at 6,8 , and $10 \mathrm{~mm}$, of all the 21 sinuses is summarized in Table 2. The MM\% ranged between 45.35 and 48.31 , with the maximum values at 8 -mm sites. The mean trend did not exactly reflect the
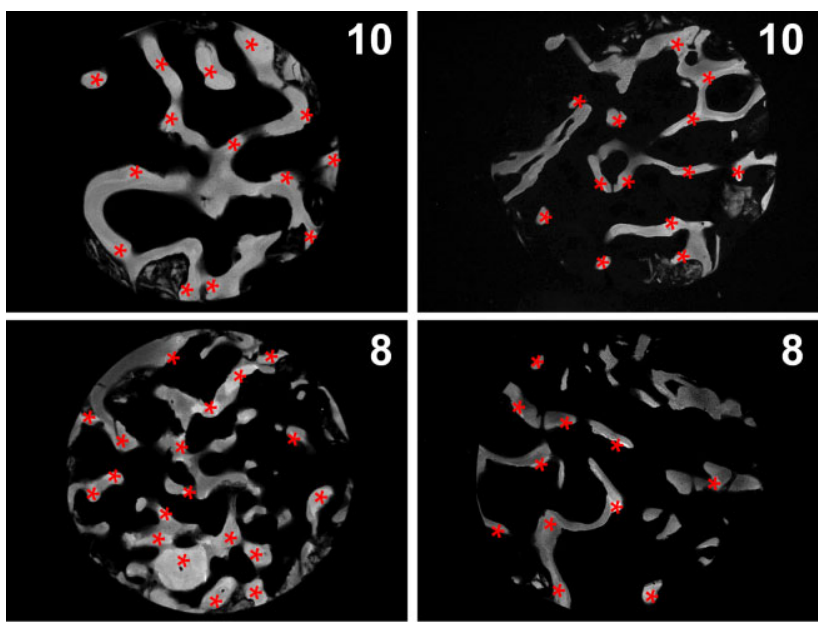

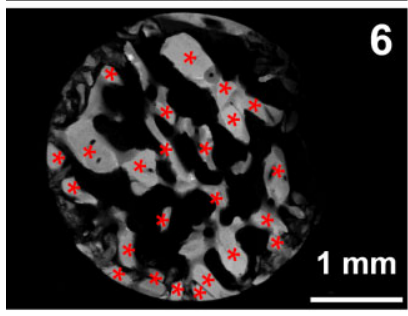

R.N.

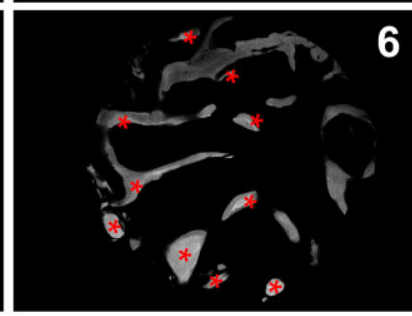

M.P.C.
Figure 2 Representative image of two-core biopsies showing the microradiographs of sections performed at the indicated level (millimeter). Note in R.N., how the mineralized material (bone and MHBA particles $[*]$ ) amount increases from the inner $(10 \mathrm{~mm})$ to the outer $(6 \mathrm{~mm})$ site, while in M.P.C., the mineralized material amount decreases from the inner to the outer site. MHBA = mineralized human bone allograft; M.P.C. $=$ initials of the patient; R.N. $=$ initials of the patient. 


\section{TABLE 2 Mineralized Material Content of} Microradiographs of the 21 Patients

\begin{tabular}{lccc}
$6 \mathrm{~mm}$ & $8 \mathrm{~mm}$ & $10 \mathrm{~mm}$ & $p(\mathrm{~K}-\mathrm{W})$ \\
\hline $45.35 \pm 15.52$ & $48.31 \pm 16.33$ & $47.29 \pm 17.62$ & .75 \\
\hline
\end{tabular}

Data are expressed as mean \pm standard deviation (percentage). $p(\mathrm{~K}-\mathrm{W})=$ probability after Kruskal-Wallis test.

individual trend: some patients showed the greater amount in the 6-mm sections, while others showed different or even opposite trends (see Figure 2). Nevertheless, no great differences existed between mean MM\% values comparing the microradiographic evaluations at 6,8 , and $10 \mathrm{~mm}$.

\section{Statistical Analysis}

Figure 3, a graphic representation of MM\% of microradiographic sections and GL of CBCT scans at implant insertion in five subjects, shows the different GL and MM\% values. Each subject had not only different values from others but also a different trend: the GL increased from the inner to the crestal side in some subjects but remained unchanged or even decreased in other patients (see Figure 3). Microradiographs showed a similar behavior with different values and trend for each patient. From the inner to the crestal side, the MM\% increased in some subjects, remained unchanged, or decreased in other subjects (see Figure 3).

The great individual differences in GL were the underlying cause of the absence of significant differences between mean GL values (see Table 1), both among "after grafting" and "at implant-insertion" sites (Kruskal-Wallis test) and between "after grafting" and "at implant-insertion" corresponding sites (Wilcoxon test for paired data). Correspondingly (see Table 2), the great individual differences in $\mathrm{MM} \%$ of microradiographic sections were the underlying cause of the absence of significant differences between sites (Kruskal-Wallis test).

Beyond GL and MM\% differences among maxillary sinus augmentations (see Figure 3 ), it was possible to assess a relationship between GL and MM\% both after Wilcoxon test (Table 3) and linear regression analysis (Figure 4). The correlation between individual GL and MM\% at 6-, 8-, and 10-mm sites resulted statistically significant $(p<.001)$ after Wilcoxon test for paired data (see Table 3). A strict correlation between the mean GL and mean $\mathrm{MM} \%$, which represent better the radiographic level of each maxillary sinus augmentation,

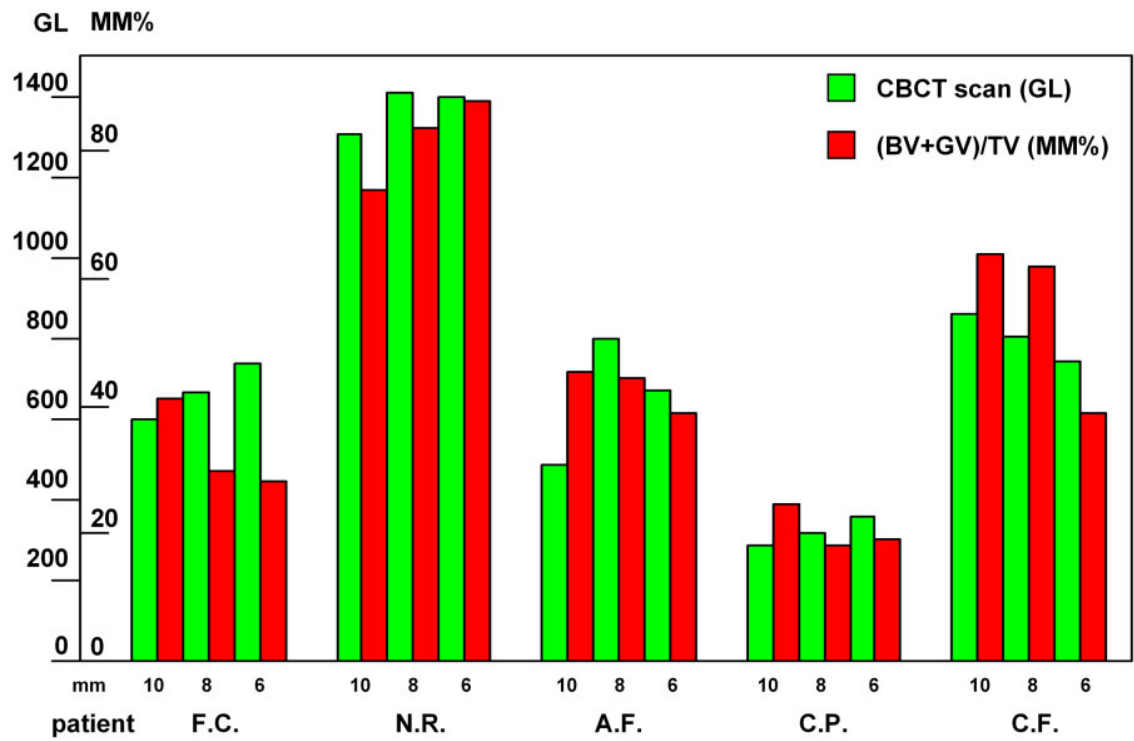

Figure 3 Representative graph showing the mean gray level (GL) of the CBCT scan and the amount of the mineralized material (MM\%) (newly formed bone and residual MHBA - [BV + GV]/TV) of the microradiograph, express as percentage (MM\%), related to the level indicated in the abscissa, in five consecutive patients. Note how the GL and MM\% value results different among patients. Furthermore, not only the trend may be individually dissimilar for GL and MM\% but also the unevenness between MM\% and GL values and trend for each patient. A.F. = initials of the patient; $[\mathrm{BV}+\mathrm{GV}] / \mathrm{TV}=($ bone volume + graft volume $) /$ tissue volume; $\mathrm{CBCT}=$ cone beam computed tomography; C.F. $=$ initials of the patient; C.P. $=$ initials of the patient; F.C. $=$ initials of the patient; $\mathrm{MHBA}=$ mineralized human bone allograft; N.R. = initials of the patient. 
TABLE 3 GLs and Mineralized Material Content of the 21 Patients at Implant Insertion

\begin{tabular}{lccc} 
& GL CBCT & $p(\mathrm{~W})$ & MM\% \\
\hline $6 \mathrm{~mm}$ & $687.2 \pm 264.0$ & $<.001$ & $45.35 \pm 15.52$ \\
$8 \mathrm{~mm}$ & $693.0 \pm 252.2$ & $<.001$ & $48.31 \pm 16.33$ \\
$10 \mathrm{~mm}$ & $646.7 \pm 282.1$ & $<.001$ & $47.29 \pm 17.62$ \\
\hline
\end{tabular}

Data are expressed as mean \pm standard deviation

$\mathrm{CBCT}=$ cone beam computed tomography; $\mathrm{GL}=$ gray level; $\mathrm{MM} \%=$ mineralized material amount; $p(\mathrm{~W})=$ probability after Wilcoxon test for paired data.

existed (see Figure 4). The simple linear regression analysis indicated that mean $\mathrm{GL}=0.0414$ (mean $\mathrm{MM} \%)+17.513$, with a correlation coefficient $r=$ 0.7707 and a consequent $p<.001$ after $t$-test.

\section{DISCUSSION}

The more accurate the diagnosis, the better the treatment should be. Nevertheless, due to the lower radiation rate and particularly in child, two-dimensional radiographs are recommended for routine preoperative dentistry. ${ }^{22}$ Dental CBCT is particularly useful or essential in pathologic condition of the jaws, ${ }^{23}$ orthodontics, ${ }^{7}$ and implant dentistry following bone regeneration. ${ }^{24}$ Although the CBCT doses are significantly lower than conventional CT, it seems reasonable to limit CBCT use due to the exposure doses, which is slightly higher than two-dimensional radiology. ${ }^{25}$

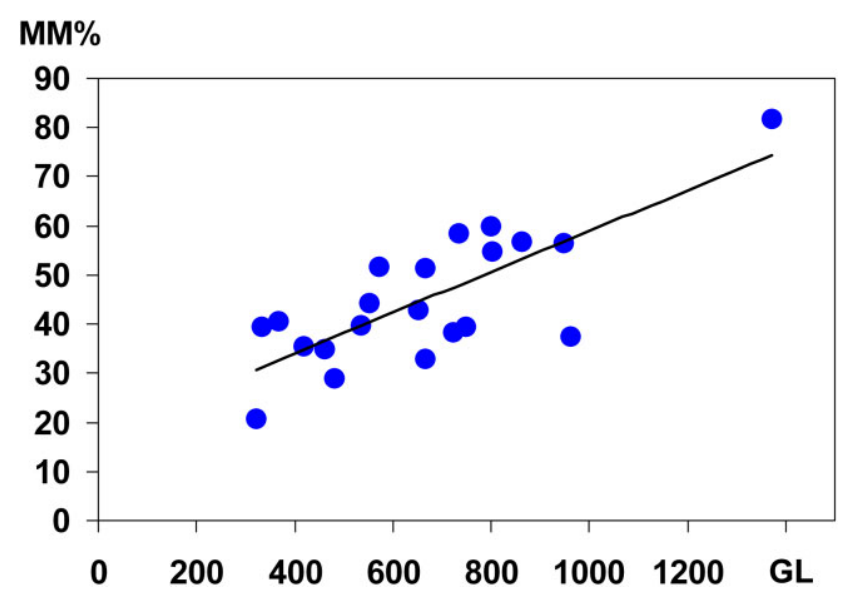

Figure 4 Relationship between the mineralized material amount (MM\%) of the microradiographs and the gray level (GL) of the 21 subjects at implant-insertion time. Each point represents the mean of 6-, 8-, and 10 -mm values. The superimposed regression line has a very good correlation coefficient $(r=0.7707)$.
CT predictability has been tested in studies on cadaveric bone, ${ }^{2,6,26}$ the relationship between densitometry and conventional histomorphometry of grafted $^{4}$ and not-grafted ${ }^{5}$ maxillae, and between densitometry and microradiograph of grafted defects. ${ }^{10}$ Most of the studies sustained the correlation between $\mathrm{CT}$ and bone amount (BV/TV), whereas a study not only did not but also disclaimed CT predictability. ${ }^{4}$

Our aim was undoubtedly challenging: to demonstrate the possibility of $\mathrm{CBCT}$ analysis to define the implant-insertion time in the dental office. Our results show that the CBCT apparatus, supplied with the suited software, can very well estimate the bone content of a site, even if the application must be improved by further studies.

Our study differed from those previously cited, ${ }^{2,4-6,11}$ not only for the use of two methods (CBCT and microradiography) employing the same physical analytic principle, the $\mathrm{x}$-rays, but also for the analysis performance in the same sites of each patient.

First, both in CBCT and microradiographs, the results showed an extremely different response in the displacement of the bone in the sites of the patients. Against all the odds, i.e., a bone amount greater in the external part and lower in the internal part of the crest, patients illustrated uniform distribution of bone amount or even an opposite behavior (greater - internal; lower - external) along the crest. This had two different explanations that singly or jointly may affect the result. We often observed some bone residues at the external extremity of core (grafted bone) biopsies. ${ }^{14}$ These are due to improper loads, such as bolus squashing, produced by the patients during the healing. This causes the external bone fragmentation and the thickening of the bone at the back by new bone formation. Second, the vascularization, which differs in each patient, may affect the bone formation and remodeling differently. Both of these activities can produce different amounts of bone, which may be at the basis of the contradictoriness or CT unpredictability found by some authors.

About the absence of a perfect parallelism between CBCT data and the BV/TV of patient sites, it must be stressed that the results are achieved using two completely different analytical systems. The microradiograph is soft x-ray radiograph of a thin section touching a photographic film. In CBCT system, the $\mathrm{x}$-rays penetrate soft and hard tissues prior to be detected by sensors, and suitable software will process the signal to 
generate each single pixel. Consequently, the CBCT analysis depends on patient characteristics, geometry, oral sites, and software capabilities and may produce some artifacts. ${ }^{27}$ Moreover, the main cause of the differences can be probably found in the unavoidable CBCT examination of the bone surrounding the implant and not that related to the core biopsy. This was due to ethical requirements, which did not allow the double CBCT scans, immediately before and after the implant placement.

On the other hand, the processing of CBCT data has an essential role in the analysis. The 3Diagnosys ${ }^{\circledR} 3.0$, medical imaging software, originates a virtual probe to extract the bone density values as GLs instead of the commonly used HUs. ${ }^{2,4-6}$ Analyzing our results, it can be stressed that no longer differences exist comparing GL and BV/TV of the three sites of each patient: a parallelism between GL values and BV/TV can be clearly seen.

The strict relationship between GL values of CBCT analysis and the BV/TV of microradiographies showed by our results fulfilled the goal of our study: demonstration predictability of the CBCT analysis. However, due to the sample size and great variations of the MM\% recorded, further studies, in particular clinical studies even joined to morphometric ones, are needed to prove that the observed bone quantity is the right amount for that particular patient, and a longer healing time can only result further bone amount decrease. ${ }^{28}$

\section{DISCLOSURE}

C.M.S.: No potential conflicts of interest to disclose. D.Z.: No potential conflicts of interest to disclose. A.M. is the technical manager of 3Diemme s.r.l. H.-L.W.: No potential conflicts of interest to disclose.

\section{ACKNOWLEDGMENTS}

This study was funded by the authors' own institutions.

\section{REFERENCES}

1. Hashizume H, Asahara H, Nishida K, Inoue H, Konishiike T. Histopathology of Kienböck's disease. Correlation with magnetic resonance and other imaging techniques. J Hand Surg [Br] 1996; 21:89-93.

2. Bousson V, Bergot C, Meunier A, et al. CT of the middiaphyseal femur: cortical bone mineral density and relation to porosity. Radiology 2000; 217:179-187.

3. Lasbleiz J, Burgun A, Marin F, Rolland Y, Duvauferrier R. Analyse de la trame osseude vertébrale sur coupes scan- ographiques [Vertebral trabecular network analysis on CT images]. J Radiol 2005; 86:645-649.

4. Schultze-Mosgau S, Keweloh M, Wiltfang J, Kessler P, Neukam FW. Histomorphometric and densitometric changes in bone volume and structure after avascular bone grafting in the extremely atrophic maxilla. Br J Oral Maxillofac Surg 2001; 39:439-447.

5. Todisco M, Trisi P. Bone mineral density and bone histomorphometry are statistically related. Int J Oral Maxillofac Implants 2005; 20:898-904.

6. Nkenke E, Hahn M, Weinzierl K, Radespiel-Tröger M, Neukam FW, Engelke K. Implant stability and histomorphometry: a correlation study in human cadavers using stepped cylinder implants. Clin Oral Implants Res 2003; 14:601-609.

7. Botticelli S, Verna C, Cattaneo PM, Heidmann J, Melsen B. Two- versus three-dimensional imaging in subjects with unerupted maxillary canines. Eur J Orthod 2011;33:344-349.

8. Arisan V, Karabuda ZC, Avsever H, Ozdemir T. Conventional multi-slice computed tomography (CT) and conebeam CT (CBCT) for computer-assisted implant placement. Part I: relationship of radiographic gray density and implant stability. Clin Implant Dent Relat Res 2012. DOI: 10.1111/ j.1708-8208.2011.00436.x.

9. Flautre B, Hardouin P. La microradiographie dans l'étude des paramètres trabéculaires [Microradiography in the study of trabecular parameters]. Acta Orthop Belg 1992; 58:287296.

10. Boivin G, Meunier PJ. The degree of mineralization of bone tissue measured by computerized quantitative contact microradiography. Calcif Tissue Int 2002; 70:503-511.

11. Nolff MC, Kokemueller H, Hauschild G, et al. Comparison of computed tomography and microradiography for graft evaluation after reconstruction of critical size bone defects using beta-tricalcium phosphate. J Craniomaxillofac Surg 2010; 38:38-46.

12. Guo S, Dipietro LA. Factors affecting wound healing. J Dent Res 2010; 89:219-229.

13. Puri KS, Suresh KR, Gogtay NJ, Thatte UM. Declaration of Helsinki, 2008: implications for stakeholders in research. J Postgrad Med 2009; 55:131-134.

14. Vercellotti T, De Paoli S, Nevins M. The piezoelectric bony window osteotomy and sinus membrane elevation: introduction of a new technique for simplification of the sinus augmentation procedure. Int J Periodontics Restorative Dent 2001; 21:561-567.

15. Stammberger H. Endoscopic endonasal surgery - concepts in treatment of recurring rhinosinusitis. Part I. Anatomic and pathophysiologic considerations. Otolaryngol Head Neck Surg 1986; 94:143-147.

16. Pikos MA. Maxillary sinus membrane repair: update on technique for large and complete perforations. Implant Dent 2008; 17:24-31. 
17. Soardi CM, Spinato S, Zaffe D, Wang HL. Atrophic maxillary floor augmentation by mineralized human bone allograft in sinuses of different size: an histologic and histomorphometric analysis. Clin Oral Implants Res 2011; 22:560-566.

18. Van Assche N, Quirynen M. Tolerance within a surgical guide. Clin Oral Implants Res 2010; 21:455458.

19. Tarnow DP, Wallace SS, Testori T, Froum SJ, Motroni A, Prasad HS. Maxillary sinus augmentation using recombinant bone morphogenetic protein-2/acellular collagen sponge in combination with a mineralized bone replacement graft: a report of three cases. Int J Periodontics Restorative Dent 2010; 30:139-149.

20. Bertoldi C, Zaffe D, Consolo U. Polylactide/polyglycolide copolymer in bone defect healing in humans. Biomaterials 2008; 29:1817-1823.

21. Glantz SA. How to test for trends (Chapter 8), and Alternatives to analysis of variance and the $t$ test based on ranks (Chapter 10). In: Glantz SA, ed. Primer of biostatistics. 5th ed. New York: Mc-Graw Hill, 2003.
22. Jung BA, Wehrbein H, Wagner W, Kunkel M. Preoperative diagnostic for palatal implants: is CT or CBCT necessary? Clin Implant Dent Relat Res 2012; 14:400-405.

23. Gahleitner A, Watzek G, Imhof H. Dental CT: imaging technique, anatomy, and pathologic conditions of the jaws. Eur Radiol 2003; 13:366-376.

24. Shibuya Y, Takeuchi Y, Asai T, Takeuchi J, Suzuki H, Komori T. Maxillary sinus floor elevation combined with a vertical onlay graft. Implant Dent 2012; 21:91-96.

25. Roberts JA, Drage NA, Davies J, Thomas DW. Effective dose from cone beam CT examinations in dentistry. Br J Radiol 2009; 82:35-40.

26. Homolka P, Beer A, Birkfellner $\mathrm{W}$, et al. Bone mineral density measurement with dental quantitative CT prior to dental implant placement in cadaver mandibles: pilot study. Radiology 2002; 224:247-252.

27. Schulze R, Heil U, Gross D, et al. Artefacts in CBCT: a review. Dentomaxillofac Radiol 2011; 40:265-273.

28. Consolo U, Zaffe D, Bertoldi C, Ceccherelli G. Platelet-rich plasma activity on maxillary sinus floor augmentation by autologous bone. Clin Oral Implants Res 2007; 18:252-262. 